\title{
Immunological detection of myeloperoxidase in synovial fluid from patients with rheumatoid arthritis
}

\author{
Steven W. EDWARDS, ${ }^{*} \ddagger$ Valerie HUGHES, ${ }^{*}$ Jill BARLOW* and Roger BUCKNALL $\dagger$ \\ *Department of Biochemistry, University of Liverpool, P.O. Box 147, and †Rheumatic Diseases Unit, \\ Royal Liverpool Hospital, Prescott Road, Liverpool L69 3BX, U.K.
}

\begin{abstract}
We have used rocket immunoelectrophoresis and immunoblotting to detect myeloperoxidase in synovial fluid from patients with rheumatoid arthritis. This protein was enzymatically inactive but its identity as myeloperoxidase was confirmed by comparing its subunit structure with that of the purified enzyme. When neutrophils were stimulated to secrete myeloperoxidase in vitro, a polypeptide with an apparent molecular mass of $62 \mathrm{kDa}$ was detected extracellularly by immunoblotting. Neutrophils isolated from synovial fluid showed a reduced level of this $62 \mathrm{kDa}$ polypeptide but it was detected extracellulary in synovial fluid by immunoblotting. Thus, we conclude that neutrophils in synovial fluid from patients with rheumatoid arthritis have been activated in vivo to secrete myeloperoxidase and propose that the products of this enzyme system can contribute to the tissue damage associated with this disease.
\end{abstract}

\section{INTRODUCTION}

Polymorphonuclear leucocytes (neutrophils) are phagocytic cells of the immune system which function to protect the host from microbial infections. In order to perform this crucial role in host defence, they contain a battery of enzymes and associated pathways which can be utilized for cell killing and these may be broadly divided into $\mathrm{O}_{2}$-independent (Elsbach \& Weiss, 1983; Spitznagel, 1984) and $\mathrm{O}_{2}$-dependent (Karnovsky \& Bolis, 1982; Babior, 1978, 1984) mechanisms. In view of the facts that neutrophils are found in large numbers in synovial fluid of patients with rheumatoid arthritis and that they have the capacity to generate oxidants extracellularly, it has been proposed that the joint damage associated with this disease is attributable, at least in part, to oxidant generation by activated neutrophils (Halliwell et al., 1985).

The generation of a full complement of reactive oxidants by neutrophils requires the activities of two enzyme systems. The first of these is an NADPH oxidase system which is responsible for the one-electron reduction of $\mathrm{O}_{2}$ to generate superoxide radicals (Babior, 1978; Rossi, 1986); $\mathrm{H}_{2} \mathrm{O}_{2}$ formation may then occur by the spontaneous or enzymatic dismutation of superoxide radicals. The second enzyme is myeloperoxidase, a haemoprotein present in azurophilic granules of neutrophils (Klebanoff \& Clark, 1978): during activation azurophilic granules release their contents at the site of oxidant generation so that myeloperoxidase can react with its substrate, $\mathrm{H}_{2} \mathrm{O}_{2}$, and thus generate $\mathrm{HOCl}$ (and related compounds) and possibly singlet $\mathrm{O}_{2}$. Formation of hydroxyl radicals $\left(\mathrm{OH}^{\circ}\right)$ may occur via reactions involving both superoxide radicals, $\mathrm{H}_{2} \mathrm{O}_{2}$ and a suitable transition metal salt catalyst, in a metal-catalysed Haber-Weiss-type reaction (Halliwell \& Gutteridge, $1985,1986)$. The high reactivities of both $\mathrm{OH}^{*}$ and $\mathrm{HOCl}$ are well documented.

It has recently been shown that iron salts in a form suitable to participate in $\mathrm{OH}^{*}$ production are detectable in some synovial fluid samples from patients with rheumatoid arthritis (Gutteridge et al., 1982; Rowley et al., 1984; Gutteridge, 1987). Therefore, considerable interest is focusing on the possible therapeutic role of iron chelators such as desferrioxamine in the treatment of this disease (Blake et al., 1985; Polson et al., 1985, 1986). However, the mere removal of transition metal salts to prevent $\mathrm{OH}^{*}$ formation will still leave the myeloperoxidase system operational and thus the possibility exists that the oxidizing products of this enzyme can evoke considerable tissue-damaging reactions. It was thus the aim of this work to establish whether neutrophils in synovial fluid from patients with rheumatoid arthritis had been activated in vivo to release myeloperoxidase in order to establish more clearly the molecular mechanisms responsible for joint degeneration in this disease.

\section{EXPERIMENTAL}

\section{Isolation and purification of cells}

Neutrophils were isolated from heparinized venous blood from healthy volunteers or patients with rheumatoid arthritis by a sedimentation procedure using $\mathbf{M}$ PRM (Flow Laboratories) exactly as described previously (Edwards et al., 1987a). Synovial fluid was collected by aspiration of knee joints and portions $(1 \mathrm{ml})$ were centrifuged at $11600 \mathrm{~g}$ for $5 \mathrm{~min}$ and the cell-free supernatants obtained were stored at $-20^{\circ} \mathrm{C}$ until use (myeloperoxidase activity was stable when stored at $-20^{\circ} \mathrm{C}$ ). The remaining synovial fluid was then diluted 2-fold with $0.9 \% \mathrm{NaCl}$, centrifuged at $1000 \mathrm{~g}$ for $5 \mathrm{~min}$ and the cell pellet was washed three times with $0.9 \%$ $\mathrm{NaCl}$. Contaminating erythrocytes were lysed by hypoosmotic lysis (Edwards \& Swan, 1986) and after restoration of tonicity, cells were centrifuged over a cushion of Ficoll-Paque (Pharmacia) at $1000 \mathrm{~g}$ for $20 \mathrm{~min}$. The purified neutrophils were washed twice and then suspended in a buffer containing (mM): $\mathrm{NaCl}, 120$; $\mathrm{KCl}, 4.8 ; \mathrm{KH}_{2} \mathrm{PO}_{4}, 1.2 ; \mathrm{CaCl}_{2}, 1.3 ; \mathrm{MgSO}_{4}, 1.2 ;$ Hepes (pH 7.4), 25; $0.1 \%$ bovine serum albumin.

‡ To whom all correspondence should be addressed. 


\section{Other methods}

Proteins were separated by polyacrylamide-gel electrophoresis in $12.5 \%$ gels containing SDS (Laemmli, 1970) and then either stained with Coomassie Blue or transferred electrophoretically to nitrocellulose (Burnette, 1981). After transfer, nitrocellulose filters were incubated in phosphate-buffered saline containing $0.2 \%$ Tween 20 and primary antibody for $1 \mathrm{~h}$ at room temperature. After washing with several changes of phosphate-buffered saline/Tween, specifically-bound primary antibody was detected by incubation with horseradish peroxidaselinked swine anti-(rabbit IgG) antibody (Dako) for $1 \mathrm{~h}$ at room temperature: after several washes the filters were stained for peroxidase using 3,3'-diaminobenzidine. Rabbit anti-(human myeloperoxidase) antibody was raised by subcutaneous injections of human myeloperoxidase (Edwards \& Swan, 1986), prepared by the method of Pember et al. (1983). Rocket immunoelectrophoresis was performed essentially as described previously (Laurell \& McKay, 1981). Myeloperoxidase was assayed enzymatically by the guaiacol method (Bergmeyer, 1974). Suitable controls indicated that oxidatively-inactivated myeloperoxidase did not have altered immuno-reactivity.

\section{RESULTS}

\section{Myeloperoxidase in synovial fluid}

Of eight samples of synovial fluid from patients with rheumatoid arthritis originally screened, only one contained detectable levels of peroxidase activity, as determined by the ability of the fluid to oxidize guaiacol to tetraguaiacol (Table 1). However, it must be stressed that many peroxidases can oxidize guaiacol and thus this single positive result cannot be unequivocally assigned to myeloperoxidase activity. We have previously shown that when myeloperoxidase is released from activated neutrophils under conditions whereby oxidants are also released, the enzyme is rapidly oxidatively inactivated (Edwards et al., 1987b) due to compound II formation (Edwards \& Lloyd, 1986). Therefore, assays designed to measure myeloperoxidase activity under these conditions will (inevitably) grossly underestimate the amount of protein present. This problem may be overcome by utilizing immunologically-based assays using anti(human myeloperoxidase) antibody. Thus, when the same synovial fluid samples were analysed for immunologically-detectable myeloperoxidase protein (rather than activity) by rocket immunoelectrophoresis, the protein was found to be present in all samples analysed, but at varying levels. Samples (analysed in triplicate) contained myeloperoxidase at levels ranging from 16-29 $\mu \mathrm{g} \cdot \mathrm{ml}^{-1}$. These results have since been confirmed in a further series of samples.

\section{Protein profiles and immunoblot analysis of synovial fluid}

In view of the fact that the synovial fluid contained immuno-reactive, but enzymatically inactive, myeloperoxidase, it was necessary to confirm the identity of this protein by analysing its molecular structure and comparing it with that of the purified enzyme.

The polypeptide profiles of the same eight synovial fluid samples analysed in Table 1 were then examined by polyacrylamide-gel electrophoresis in order to identify

\section{Table 1. Myeloperoxidase in synovial fluid}

Portions of synovial fluid from patients with rheumatoid arthritis (after removal of cells) were assayed for peroxidase by its ability to oxidize guaiacol to tetraguaiacol. The samples were then assayed for myeloperoxidase protein by rocket immunoelectrophoresis and quantified by comparing rocket areas with those obtained using dilutions of purified enzyme. N.D., non detected.

\begin{tabular}{ccc}
\hline Sample & $\begin{array}{c}\text { Enzyme } \\
\text { activity } \\
\text { (munits/ml) } \\
\text { (guaiacol } \\
\text { oxidation) }\end{array}$ & $\begin{array}{c}\text { Amount of protein } \\
(\mu \mathrm{g} / \mathrm{ml} \text { ) (rocket } \\
\text { immunoelectrophoresis) }\end{array}$ \\
\hline & N.D. & 16 \\
1 & N.D. & 19 \\
2 & N.D. & 17 \\
3 & N.D. & 29 \\
4 & N.D. & 18 \\
5 & H.2 & 23 \\
6 & N.D. & 20 \\
7 & N.D. & 20 \\
8 &
\end{tabular}

polypeptide(s) which could be attributable to purified myeloperoxidase. The major polypeptides of synovial fluid had apparent molecular masses of $61-63,57$ and $51-53 \mathrm{kDa}$ and only minor differences in polypeptide profiles and intensity of staining were observed in the different samples (Fig. 1a). The major subunit of purified myeloperoxidase in this range was $55 \mathrm{kDa}$ (with lesswell-defined bands at $39 \mathrm{kDa}$ and $15 \mathrm{kDa}$ ).

Identical samples were then immunoblotted and myeloperoxidase visualized using primary antibody raised to the purified enzyme. Purified myeloperoxidase comprised two immuno-reactive subunits of apparent molecular masses of 55 and $63 \mathrm{kDa}$ and all eight synovial fluid samples contained a polypeptide of $63 \mathrm{kDa}$ which cross-reacted with the antiserum (Fig. $1 b$ ).

\section{Polypeptide profiles and immunoblots of neutrophils stimulated in vitro}

Neutrophils may be activated in vitro by the chemotactic peptide fMet-Leu-Phe plus cytochalasin B to secrete myeloperoxidase (Edwards et al., 1987b). Therefore, the polypeptide profiles of non-activated and activated cells, together with released proteins, were compared and myeloperoxidase identified by immunoblotting. The polypeptide profiles of non-activated and activated cells were remarkably similar but in cells activated to secrete myeloperoxidase, polypeptides with apparent molecular masses of $74,63,55$ and $39 \mathrm{kDa}$ stained less intensely (Fig. 2a). Correspondingly, polypeptides with apparent molecular masses of 74, 63, 55 and $39 \mathrm{kDa}$ (plus a few other bands) were found extracellularly after activation (Fig. $2 a$ ).

Identical samples were then immunoblotted and stained for immuno-detectable myeloperoxidase. Purified myeloperoxidase comprised immuno-reactive polypeptides with apparent molecular masses of 55 and $63 \mathrm{kDa}$ while in both activated and non-activated neutrophils the same three polypeptides could also be distinguished, the $63 \mathrm{kDa}$ band staining most intensely (Fig. 2b). In the cell-free supernatant from activated cells the $63 \mathrm{kDa}$ polypeptide was clearly identified (Fig. $2 b$ ). 
(a)

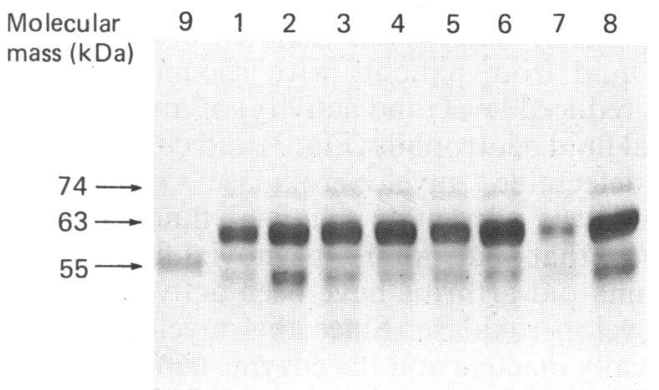

(b)

$\begin{array}{lllllllllllll}\text { Lane } & 8 & 7 & 6 & 5 & 4 & 3 & 2 & 1 & 9 & \begin{array}{l}\text { Molecular } \\ \text { mass (kDa) }\end{array}\end{array}$

Fig. 1. Protein profiles and immunoblots of synovial fluid

Samples of synovial fluid $(1 \mu \mathrm{l})$ were solubilized in SDS-sample buffer containing $\beta$-mercaptoethanol (99 $\mu \mathrm{l})$ and heated to $90^{\circ} \mathrm{C}$ for $2 \mathrm{~min}$. Two $12.5 \%$ polyacrylamide gels were prepared and to one of these $25 \mu 1$ of the above mix was added (lanes 1-8). After electrophoresis this gel was stained for protein using Coomassie Blue $(a)$. To the second gel, $10 \mu \mathrm{l}$ of the above mix was added and after electrophoresis was immunoblotted $(b)$. Purified myeloperoxidase $(5 \mu \mathrm{g}$, lane 9$)$ was loaded to the protein gel and $1 \mu \mathrm{g}$ to the gel which was immunoblotted. Apparent molecular masses were calculated by comparing the relative mobilities with those of standards (Sigma, SDS-7).

(a)

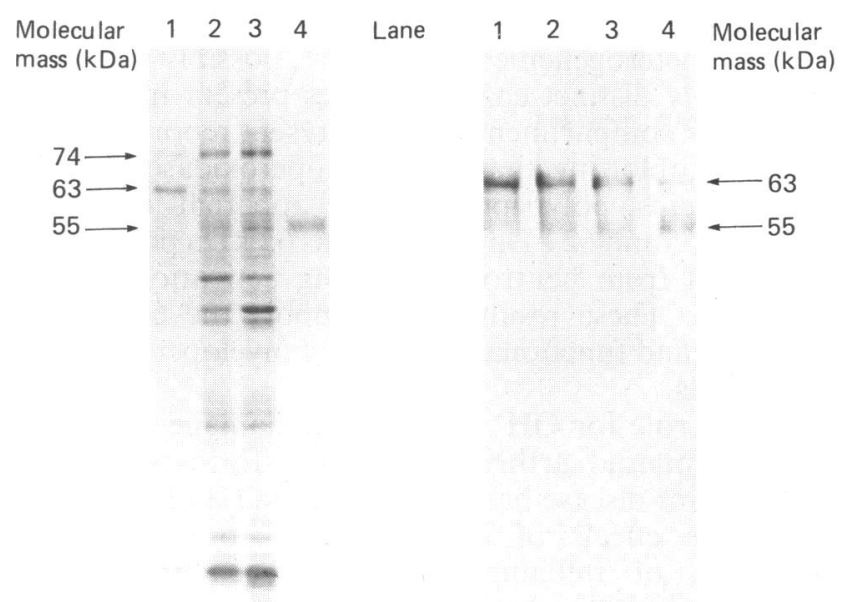

Fig. 2. Protein profiles and immunoblots of neutrophils activated in vitro

Neutrophils were purified from the peripheral blood of healthy volunteers and suspended in buffer devoid of bovine serum albumin. Two portions containing $2 \times 10^{6}$ cells were taken and to one of these $1 \mu \mathrm{M}$-f Met-Leu-Phe plus $1 \mu \mathrm{g}$ of cytochalasin $\mathrm{B} / \mathrm{ml}$ (final concns.) was added. After incubation at $37^{\circ} \mathrm{C}$ for $5 \mathrm{~min}$ both this and the unstimulated suspension were centrifuged for $2 \mathrm{~min}$ at high speed in an MSE Microcentaur and the cell pellets and supernatants retained. The cell pellets were resuspended in $900 \mu \mathrm{l}$ of water and proteins were precipitated
Polypeptide profiles and immunoblots of neutrophils and synovial fluid from patients with rheumatoid arthritis

Since it was shown (Fig. 2) that neutrophils which had been activated in vitro secrete myeloperoxidase and that this was identified extracellularly, it was then necessary to determine whether this process also occurred during activation in vivo in patients with rheumatoid arthritis. Therefore, neutrophils were prepared from the synovial fluid and peripheral blood of a patient with rheumatoid arthritis and the polypeptides were compared together with the synovial fluid itself. The polypeptide profiles of synovial fluid neutrophils and bloodstream neutrophils were remarkably similar except that in the former, polypeptides with apparent molecular masses of 74 and $55 \mathrm{kDa}$ (plus a few other polypeptides) stained less intensely than in bloodstream neutrophils (Fig. $3 a$ ). Corresponding immunoblots revealed purified myelo-

from these and the cell-free supernatant by adding trichloroacetic acid to $10 \%$ followed by incubation at $4{ }^{\circ} \mathrm{C}$ for $16 \mathrm{~h}$. The precipitates were then centrifuged and pellets washed five times with ether: after the final wash residual traces of ether were removed by warming to $37^{\circ} \mathrm{C}$. Precipitates were then dissolved in SDS-sample buffer containing $\beta$-mercaptoethanol and $25 \mu \mathrm{l}$ of this mixture loaded for protein staining (a) or $10 \mu \mathrm{l}$ loaded for immunoblotting $(b)$. Lane 1 , supernatant from activated neutrophil suspensions; 2 , cell extract from stimulated neutrophils; 3 , cell extract from unstimulated neutrophils; 4 , purified myeloperoxidase $(5 \mu \mathrm{g}$ in $a, 1 \mu \mathrm{g}$ in $b)$. No proteins were detected in cell free supernatants from nonstimulated neutrophils. 


\begin{tabular}{llllllllll} 
& \multicolumn{1}{c}{ (a) } & & \multicolumn{3}{c}{$(b)$} & & \\
& & & & & & & & & \\
$\begin{array}{l}\text { Molecular } \\
\text { mass }(\mathrm{kDa})\end{array}$ & 1 & 2 & 3 & Lane & 3 & 1 & 2 & 4 & $\begin{array}{l}\text { Molecular } \\
\text { mass }(\mathrm{kDa})\end{array}$
\end{tabular}

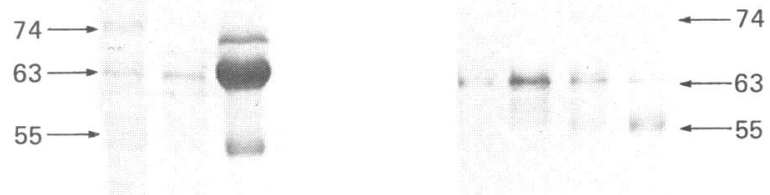

Fig. 3. Protein profiles and immunoblots from neutrophils and synovial fluid from a patient with rheumatoid arthritis

Neutrophils were prepared from venous blood and synovial fluid from a patient with rheumatoid arthritis. Samples of neutrophils $\left(2 \times 10^{6}\right.$ cells $)$, synovial fluid and myeloperoxidase were prepared and loaded as described in the legends to Figs. 1 and 2. (a) Protein stained; $(b)$ immunoblots. Lane 1, cell extract from bloodstream neutrophils ; 2 , cell extract from synovial fluid neutrophils; 3 , synovial fluid; 4 , purified myeloperoxidase.

peroxidase subunits with apparent molecular masses of 55,63 (and 74) $\mathrm{kDa}$ and that the 63 and to a lesser extent the $55 \mathrm{kDa}$ polypeptides were identified in bloodstream neutrophils (Fig. $3 b$ ). However, in neutrophils isolated from the synovial fluid from the same patient, the $63 \mathrm{kDa}$ subunit stained less intensely than in bloodstream neutrophils and correspondingly this polypeptide was found extracellularly in the synovial fluid (Fig. $3 b$ ). Essentially the same results were obtained when similar experiments were repeated using samples prepared from a further three patients. In these samples (and in samples from a further three patients) the total myeloperoxidase activity in synovial fluid neutrophils was only $15-20 \%$ of that present in corresponding bloodstream neutrophils from the same patients.

\section{DISCUSSION}

The results presented here clearly show that synovial fluid from patients with rheumatoid arthritis contain immunologically-detectable, but enzymatically-inactive myeloperoxidase; this protein was quantified by rocket immunoelectrophoresis and its identity as myeloperoxidase was confirmed by comparing immunoblots with those obtained for the purified enzyme. Bloodstream neutrophils may be activated in vitro to secrete myelo- peroxidase (Edwards et al., 1987b) and polypeptides cross-reacting with anti-(myeloperoxidase) antiserum were detected extracellularly (Fig. 2). Comparison of immunoblots of neutrophils isolated from blood and synovial fluid from patients with rheumatoid arthritis showed a reduced level (and activity) of myeloperoxidase in synovial fluid neutrophils (Fig. 3) and correspondingly, immuno-detectable myeloperoxidase released from neutrophils was present in synovial fluid. Thus, these results show that neutrophils in synovial fluid of patients with rheumatoid arthritis have been activated in vivo to secrete myeloperoxidase. Since this myeloperoxidase is enzymatically inactive and the enzyme can be oxidatively inactivated in vitro (Edwards et al., 1987b), it is therefore proposed that in synovial fluid myeloperoxidase has been secreted concomitantly with activation of oxidant generation, although more direct evidence may be required to clarify this latter point.

Much controversy exists in the literature regarding the subunit structure of purified myeloperoxidase, although molecular cloning techniques should help clarify this apparent complexity and confusion (Chang et al., 1986; Johnson et al., 1987; Morishita et al., 1987; Weil et al., 1987). For example, the purified enzyme from human neutrophils or HL-60 cells has been reported to comprise subunits of 55-63 and 10-15 kDa and also a $39 \mathrm{kDa}$ subunit in non- or partially-reduced preparations (Matheson et al., 1981; Olsen \& Little, 1984; Olsson et al., 1984; Koeffler et al., 1985; Nauseef, 1986; Nauseef \& Malech, 1986). The enzyme is synthesized as a single, large precursor and is then cleaved to yield a number of intermediary polypeptides one of which has a molecular mass of $74 \mathrm{kDa}$ (Koeffler et al., 1985; Akin \& Kinkade, 1986). Only a few reports have described preparations containing more than one subunit in the range 55-63 kDa (Pember et al., 1983; Akin \& Kinkade, 1986; Miyasaki et al., 1986) but these have been proposed to represent heterogeneities in structure, possibly indicating functionally distinct enzyme species present in separate subcellular compartments. In the present report we show by immunoblotting that our myeloperoxidase preparation contains polypeptides with apparent molecular masses of 55 and $62 \mathrm{kDa}$ and that this $62 \mathrm{kDa}$ polypeptide is secreted from neutrophils during activation in vitro or in vivo. These results thus support the concept of structural and functional diversity of myeloperoxidase in neutrophils.

Since a role for $\mathrm{OH}^{*}$ in the tissue damage associated with rheumatoid arthritis and other forms of inflammatory joint disease has been proposed (Halliwell et al., 1985), the effects of iron chelators to alleviate the symptoms of inflammation have been investigated (Blake et al., 1983; Andrews et al., 1987). However, the activity of myeloperoxidase and its ability to generate reactive oxidants such as $\mathrm{HOCl}$ is independent of the level of iron in its environment. Thus, therapeutic regimes designed to restrict available iron levels will limit $\mathrm{OH}^{\circ}$ production, but will not affect oxidant generation by myeloperoxidase. Further work is therefore necessary to evaluate the role of myeloperoxidase products in inflammatory joint disease and it is of note that many antiarthritis drugs currently in use have been shown to scavenge the myeloperoxidase product, $\mathrm{HOCl}$ (Matheson, 1982; Cuperus et al., 1985). Further work is also necessary to correlate the levels of myeloperoxidase released into synovial fluid with the severity of the 
disease, in order to fully assess the clinical significance of these observations. Since no specific inhibitor for myeloperoxidase exists (Edwards \& Swan, 1986), a supplementary approach to the use of iron chelators in rheumatoid arthritis will now be to search for specific agents which selectively inhibit myeloperoxidase.

We thank the Arthritis and Rheumatism Council for generous financial support.

\section{REFERENCES}

Akin, D. T. \& Kinkade, J. M., Jr. (1986) J. Biol. Chem. 261, $8370-8375$

Andrews, F. J., Morris, C. J., Kondratowicz, G. \& Blake, D. R. (1987) Ann. Rheum. Dis. 46, 327-333

Babior, B. M. (1978) N. Engl. J. Med. 298, 659-668

Babior, B. M. (1984) Blood 64, 959-966

Bergmeyer, H. U. (ed.) (1974) Methods of Enzymatic Analysis, 2nd Edition, pp. 685-690, Verlag Chemie, Weinheim

Blake, D. R., Hall, N. D., Bacon, P. A., Dieppe, P. A., Halliwell, B. \& Gutteridge, J. M. C. (1983) Ann. Rheum. Dis. 42, 89-93

Blake, D. R., Winyard, P., Lunec, J., Williams, A., Good, P. A., Gutteridge, J. M. C., Rowley, D. \& Halliwell, B. (1985) Quart. J. Med. 56, 345-355

Burnette, W. N. (1981) Anal. Biochem. 112, 195-203

Chang, K. S., Trujillo, J. M., Cook, R. G. \& Stass, S. A. (1986) Blood 68, 1411-1414

Cuperus, R. A., Muijsers, A. O. \& Wever, R. (1985) Arthritis Rheum. 28, 1228-1233

Edwards, S. W. \& Lloyd, D. (1986) Biosci. Rep. 6, 275-282

Edwards, S. W. \& Swan, T. F. (1986) Biochem. J. 237, 601-604

Edwards, S. W., Say, J. E. \& Hart, C. A. (1987a) J. Gen. Microbiol. 133, in the press

Edwards, S. W., Nurcombe, H. L. \& Hart, C. A. (1987b) Biochem. J. 245, 925-928

Elsbach, P. \& Weiss, J. (1983) Rev. Infect. Dis. 5, 843-853

Gutteridge, J. M. C. (1987) Biochem. J. 245, 415-421

Gutteridge, J. M. C., Rowley, D. A. \& Halliwell, B. (1982) Biochem. J. 206, 605-609

Halliwell, B. \& Gutteridge, J. M. C. (1985) Arch. Biochem. Biophys. 246, 501-514

Received 3 August 1987/24 September 1987; accepted 12 October 1987
Halliwell, B., Gutteridge, J. M. C. \& Blake, D. (1985) Philos. Trans. R. Soc. London Ser. B 311, 659-671

Halliwell, B. \& Gutteridge, J. M. C. (1986) Molec. Aspects Med. 8, 89-193

Johnson, K. R., Nauseef, W. M., Care, A., Wheelock, M. J., Shane, S., Hudson, S., Koeffler, H. P., Selsted, M., Miller, C. \& Rovera, G. (1987) Nucleic Acids Res. 15, 2013-2028

Karnovsky, M. L. \& Bolis, L. (eds.) (1982) Phagocytosis-Past and Future, p. 592, Academic Press, London and New York

Klebanoff, S. J. \& Clark, R. A. (1978) The Neutrophil: Function and Clinical Disorders, p. 810, North-Holland, Amsterdam

Koeffler, H. P., Ranyard, J. \& Pertcheck, M. (1985) Blood 65, 484-491

Laemmli, U. K. (1970) Nature (London) 227, 680-685

Laurell, C.-B. \& McKay, E. J. (1981) Methods Enzymol. 73, 339-383

Matheson, N. R. (1982) Biochem. Biophys. Res. Commun. 108, 259-265

Matheson, N. R., Wong, P. S. \& Travis, J. (1981) Biochemistry 20, 325-330

Morishita, K., Kubota, N., Asano, S., Kaziro, Y. \& Nagata, S. (1987) J. Biol. Chem. 262, 3844-3851

Miyasaki, K. T., Wilson, M. E., Cohen, E., Jones, P. C. \& Genco, R. J. (1986) Arch. Biochem. Biophys. 246, 751-764

Nauseef, W. M. (1986) Blood 67, 865-872

Nauseef, W. M. \& Malech, H. L. (1986) Blood 67, 1504-1507

Olsen, R. L. \& Little, C. (1984) Biochem. J. 222, 701-709

Olsson, I., Persson, A.-M. \& Stömberg, K. (1984) Biochem. J. 223, 911-920

Pember, S. O., Shapira, R. \& Kinkade, J. M., Jr. (1983) Arch. Biochem. Biophys. 221, 391-403

Polson, R. J., Jawad, A., Bomford, A., Berry, H. \& Williams, R. (1985) Br. Med. J. 291, 448

Polson, R. J., Jawad, A. S. M., Bomford, A., Berry, H. \& Williams, R. (1986) Q. J. Med. 236, 1153-1158

Rossi, F. (1986) Biochim. Biophys. Acta 853, 65-89

Rowley, D., Gutteridge, J. M. C., Blake, D. R., Farr, M. \& Halliwell, B. (1984) Clin. Sci. 66, 691-695

Spitznagel, J. K. (1984) in Contemporary Topics in Immunology: Regulation of Leukocyte Function (Snyderman, R., ed.), vol. 14, pp. 283-343, Plenum Press, New York

Weil, S. C., Rosner, G. L., Reid, M. S., Chisholm, R. L., Farber, N. M., Spitznagel, J. K. \& Swanson, M. S. (1987) Proc. Natl. Acad. Sci. U.S.A. 84, 2057-2061 